\title{
Improved Diagnosis of Systemic Sclerosis Using Nailfold Capillary Flow
}

DOI:

10.1007/978-3-319-46726-9_40

\section{Document Version}

Accepted author manuscript

Link to publication record in Manchester Research Explorer

\section{Citation for published version (APA):}

Berks, M., Dinsdale, G., Murray, A., Moore, T., Herrick, A., \& Taylor, C. (2016). Improved Diagnosis of Systemic Sclerosis Using Nailfold Capillary Flow. In S. Ourselin, L. Joskowicz, M. R. Sabuncu, G. Unal, \& W. Wells (Eds.), Medical image computing and computer-assisted intervention -- MICCAI 2016 : 19th International Conference, Athens, Greece, October 17-21, 2016, Proceedings. (Vol. 3, pp. 344-352). (Lecture Notes in Computer Science; Vol. 9902). Springer Nature. https://doi.org/10.1007/978-3-319-46726-9_40

\section{Published in:}

Medical image computing and computer-assisted intervention -- MICCAI 2016 : 19th International Conference, Athens, Greece, October 17-21, 2016, Proceedings.

\section{Citing this paper}

Please note that where the full-text provided on Manchester Research Explorer is the Author Accepted Manuscript or Proof version this may differ from the final Published version. If citing, it is advised that you check and use the publisher's definitive version.

\section{General rights}

Copyright and moral rights for the publications made accessible in the Research Explorer are retained by the authors and/or other copyright owners and it is a condition of accessing publications that users recognise and abide by the legal requirements associated with these rights.

\section{Takedown policy}

If you believe that this document breaches copyright please refer to the University of Manchester's Takedown Procedures [http://man.ac.uk/04Y6Bo] or contact uml.scholarlycommunications@manchester.ac.uk providing relevant details, so we can investigate your claim.

\section{OPEN ACCESS}




\title{
Improved Diagnosis of Systemic Sclerosis using Nailfold Capillary Flow
}

\author{
Michael Berks, Graham Dinsdale, Andrea Murray, Tonia Moore, Ariane Herrick, and \\ Chris Taylor \\ University of Manchester, Manchester, United Kingdom \\ michael.berks@manchester.ac.uk
}

\begin{abstract}
Nailfold capillaroscopy (NC) allows non-invasive imaging of systemic sclerosis (SSc) related microvascular disease. We have developed a state-of-theart NC system that enables fast, panoramic imaging of the whole nailfold at highmagnification, and incorporates novel software to make fully automated estimates of capillary structure and blood flow velocity. We present the first results of a study in which 50 patients with SSc, 12 with primary Raynauds phenomenon (PRP) and 50 healthy controls (HC) were imaged using the new system, and show that a combined model of capillary measurements strongly separates SSc from $\mathrm{HC} / \mathrm{PRP}$ (ROC $A_{z}=0.93$ ). Including capillary flow improves model performance, suggesting flow provides complementary information to capillary structure for diagnosing SSc.
\end{abstract}

\section{Introduction}

Nailfold capillaroscopy (NC) is a non-invasive optical technique for imaging microvascular abnormalities, commonly used in the diagnosis and assessment of systemic sclerosis (SSc) - an automimmune connective tissue disease that is painful, disabling and disfiguring, with high mortality. In particular, NC can identify the characteristic structural changes in capillaries that differentiate patients with symptoms of SSc induced Raynauds phenomenon (RP; the most common presenting feature of SSc) from those with the more common and relatively benign primary (idiopathic) RP (PRP) [1, 2].

In current clinical practice, such changes are labelled as either normal/abnormal or divided into coarse disease stages (early, active, late). However subjective labelling suffers from poor inter-observer agreement, and is not well-suited for monitoring disease progression. With the development of drugs with vascular remodelling potential, and increasing interest in early intervention, there has been a move towards quantitative assessment [3,4]. Manually measuring capillary size, shape and density is labour intensive, but has been shown to be more reproducible than qualitative assessment [3], and shows good discrimination between SSc and PRP. Recent work has shown such measures can be made automatically to the same level of accuracy as expert observers [4].

However, these structural changes happen on a timescale of months/years and may not be useful for monitoring rapid changes, e.g. in a clinical trial of vasoactive therapy. In contrast, capillary blood flow may respond immediately to such interventions, whilst also providing complementary information to structural measures in routine diagnosis of SSc. Moreover, flow, as a measure of capillary function, can potentially provide 
insights into pathophysiology and markers of disease activity, both in SSc and other conditions in which the microvasculature plays a key role.

Motivated by these observations, we have developed a state-of-the-art NC system that uses a high frame rate camera to capture video sequences in which it is possible to measure red blood cell velocity in individual capillaries. The system includes software to generate high-quality static image mosaics for clinical assessment, and makes fullyautomated measures of capillary structure and flow - the latter using a novel adaption of optimised optical flow [5] developed to be robust to the extremely challenging lowcontrast, high noise conditions inherent in $\mathrm{NC}$ imaging.

While flow has been estimated previously in NC video [6,7], this was performed only at manually selected points or vessels, an approach that both introduces subjectivity and does not scale to analysing large datasets. We believe our system is the first to measure flow fully-automatically in all visible capillaries across the whole nailfold. Capillary blood flow has also been measured using the related technique of sidestream dark field (SDF) imaging (e.g. in [8] amongst others), however SDF requires skin contact and may be unsuitable for assessing patients with SSc.

In this paper we describe the new system, and present the first results of a trial in which it was used to image 50 healthy controls (HC), 12 patients with PRP and 50 patients with SSc. We show that automated measures of capillary structure can differentiate patients with SSc from HC/PRP providing further evidence of the validity of the technique, that a combined model provides greater predictive power than individual measures, and that including capillary blood velocity improves prediction, suggesting capillary flow can aid the diagnosis of SSc.

\section{A New High Frame Rate Capillaroscopy System}

The new system comprises a monochrome, high frame rate digital camera (The Imaging Source, DTK23U618, 640x480 px, 120fps) attached to a $27 \mathrm{~mm}$ focal length, $9 \mathrm{~mm}$ diameter lens, situated $152 \mathrm{~mm}$ from the camera sensor to produce raw video frames with a spatial resolution of $1 \mu \mathrm{m}$ per pixel. The camera is connected to a 3-D highprecision motorised stage system (Thorlabs, MTS25/M-Z8) with $25 \mathrm{~mm}$ of travel along each axis. This enables fast and accurate focusing and imaging mosaicking so the whole nailfold can be imaged at high-magnification. For patients unable to straighten their fingers (a common disability in SSc), the stages are mounted to a stand that can be rotated through $\sim 75^{\circ}$. The system thus combines the flexibility of a hand-held capillaroscopy device with the advantages of a rigid platform for obtaining high-quality images ${ }^{1}$.

\subsection{Acquiring images}

To complement the hardware, we have developed a complete software package for acquiring image video sequences and recording associated session data. During acquisition, live video from the camera is displayed alongside a map showing the cameras current 3 -D position within the $25 \mathrm{~mm}^{3}$ travel permitted by the motors. The camera can

\footnotetext{
${ }^{1}$ Videos available at http://personalpages.manchester.ac.uk/staff/Michael.Berks/nailfolddemo
} 
(a)

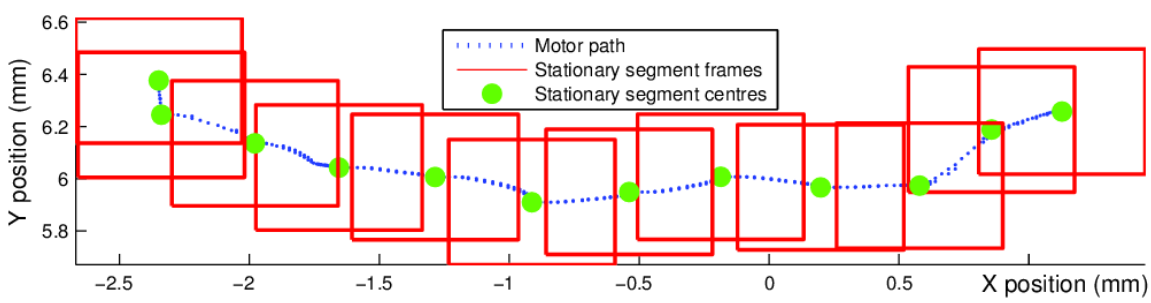

(b)

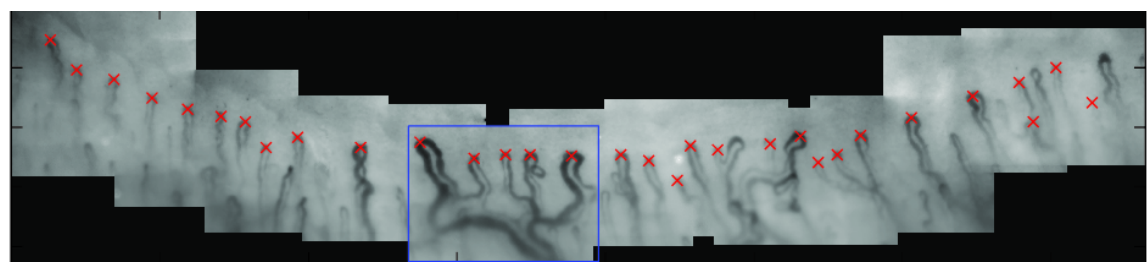

Fig. 1. Generating mosaics (a) video sequence motor positions are used to compute stationary segments and estimate the mosaic's global geometry; (b) registered mosaic, with the distal capillaries detected. The blue box marks a stationary segment from which capillary videos are extracted.

be moved to any $(x, y)$ position, either by clicking or dragging a joystick in the live display (for precision movements) or clicking in the map (for fast long range movements). Focus is controlled by moving the cameras in the $z$-plane using either keyboard arrows or the mouse scroll-wheel. During recording each frame is tagged with the current motor position and every 30th frame is added to a live image mosaic, registered in real-time to show the user the extent of the nailfold captured ${ }^{1}$.

\subsection{Generating mosaics and capillary videos}

A complete video sequence of a nailfold typically comprises 5,000-15,000 raw frames, which are processed offline to generate high-quality static image mosaics and video sequences of each visible capillary. Consecutive frames can be separated into 3 types of segment where: 1$)$ the motors are moving in the (x,y)-plane (panning); 2) the $\mathrm{z}$-motor is moving (focusing); 3 ) the motors are stationary. The acquistion software limits continuous panning to guarantee that stationary segments overlap, and only these frames are used to analyse capillaries (Fig. 1a).

In the first processing step, frames within each segment are aligned and saved for later use in capillary videos (Section 4). The aligned frames are averaged to form a compound frame with significantly lower noise and better contrast than the raw frames [9]. To form a mosaic of the whole nailfold, the compound frames are then sequentially registered by searching for the global best match of vessel edge features, generated by non-maximally suppressing the response to a Gaussian1st derivative filter. Frames are initially aligned using their motor position (Fig. 1a). To guard against mis-registering frames with very little or no image content (a problem inherent in some nailfolds, both due to low-contrast and because disease may have destroyed capillary structure), frames that do not meet a minimum matching threshold are kept in their initial position, preventing total registration failure observed in earlier NC systems that employed mosaick- 
ing [9]. This is particularly important where capillaries are only present/visible in some regions of a nailfold, as by keeping the global geometry of the mosaic intact, we can still safely measure capillary density.

\section{Measuring capillary structure}

Having generated an image mosaic of the whole nailfold we use the method described by Berks et al. [4] to detect the distal row of capillaries (Fig. 1b). The method first predicts pixel level estimates of vesselness $\left(V_{s}\right.$ i.e. the probability of belonging to a vessel), vessel orientation $\left(V_{\theta}\right)$ and width $\left(V_{w}\right)$, as outputs from random forests trained on manually labelled capillaries, with features based on a multi-scale, multi-orientation decomposition of local image strcuture. The pixel level maps are used to apply a second level of machine learning in which scaled and rotated image patches are extracted from candidate vessel centres and used to predict the location of nearby capillary apices. A final selection of distal row capillaries can then be made using both local image appearance and the spatial relationships between the candidate apices.

As in [4] we use the location of each distal capillary apex to compute capillary density (defined as the distance between the leftmost and rightmost distal capillaries, divided by the number of distal capillaries). In addition, using $V_{s}$ we compute the set of vessel pixels connected to, and at most $100 \mu \mathrm{m}$ from the apex of each capillary, and subsequently use $V_{w}$ and $V_{\theta}$ to compute the mean width $\bar{W}$ and orientation $\bar{\Theta} . \bar{W}$ is as a more robust measure of indiviudal capillary width than a point estimate at the apex and is used to compute mean and maximum widths of all capillaries in the nailfold.

Meanwhile, $\bar{\Theta}$, is used to compute two further measures of structure: shape and derangement. Pixel orientations in $V_{\theta}$ are represented as angle-doubled unit vectors in the complex plane, $V_{\theta}(p)=\exp 2 i \phi$. Thus for each capillary we can write $\bar{\Theta}$ as a complex number $S \exp 2 i \bar{\phi}$ where the magnitude $S \in[0,1]$ is a measure of circular dispersion that will be higher in normal vessels (with long straight limbs and a narrow hairpin apex) than abnormally shaped capillaries. The mean of each individual capillary dispersion $S$ in the image gives a measure of shape uniformity for the whole nailfold.

Returning to $\bar{\Theta}$, the angle $\bar{\phi}$ represents the principal direction of the capillary. Discarding $S$, and taking the average of the unit vectors $\hat{\Theta}=\exp 2 i \bar{\phi}$ across the nailfold generates a new mean orientation, the dispersion parameter of which $\bar{D}$ provides a measure of the uniformity of capillary direction (derangement), again with range $[0,1]$ and likely to be higher in nailfolds with normal structure.

The final set of structural measurements for each nailfold comprise capillary density, mean and maximum width, shape and derangement, approximately corresponding to measures made in earlier (semi-)manual quantitative analysis [3].

\section{Measuring capillary flow}

To build videos of capillary blood flow, we use the location of detected capillaries and the regsitered position of each compound frame in the nailfold mosaic to select the distal row capillaries visible in each stationary segment (Fig. 2a). For a given segment, 
(a)

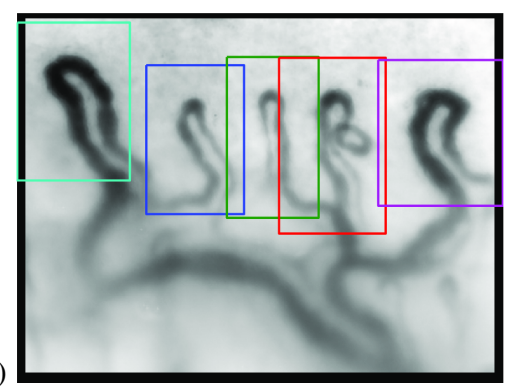

(b)

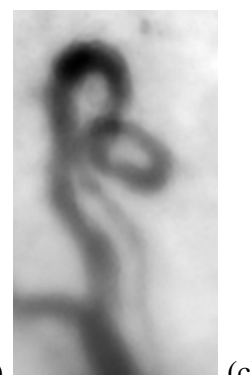

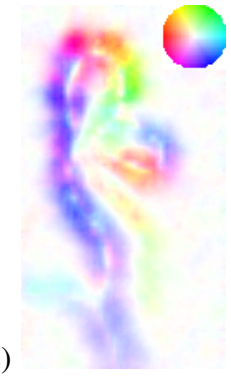

Fig. 2. Measuring capillary flow: (a) capillary bounding boxes in a stationary segment; (b) average of re-registered cropped frames from the red box ${ }^{1}$; (c) Estimate of vessel flow-colour denotes flow direction, intensity flow magnitude.

we use $V_{s}$ to define a bounding box around the extremeties of each capillary, and use this to sample patches from the segment's frames.

The sampled patches for each capillary are re-registered using the compound frame, cropped to the capillary's bounding box, as a common target. This corrects pixel-level mis-registrations in the global frame alignment that make little difference when averaged in the static image, but significantly degrade flow estimation. Finally the registered patches are contrast normalised ready for flow estimation (Fig. 2b).

To estimate blood velocity in a capillary video, we use a multiscale version of optical flow, based on the work of Brox et al. [5]. First, we form a pyramid stack of the patches, successively smoothing and downsampling the patches at each pyramid level. We then use least-squares non-linear optimisation to compute a dense flow field in the coarsest layer. This flow field is upsampled and used to warp the patches at the next pyramid level, before recomputing spatio-temporal gradients, and optimising again to find the new flow field. The process repeats until we reach the original image resolution.

The non-linear least-squares optimisation at each level combines a data term (that the pixels should obey the standard optical flow equations and thus maintain constant brightness [10]) coupled with a smoothness term that acts to spatialy regularise the flow field (that nearby locations should have similar flow) while permitting non-linearities at object boundaries. We assume that flow is constant over time at any given location for the duration of the video, computing a single flow vector that optimally describes the observed horizontal and vertical displacements. At the finest pyramid level this effectively computes the mean flow over time at each pixel (Fig. 2c). Measuring flow in this way is considerably more robust to noise than estimating flow between pairs of frames, and is necessary to cope with the high-noise, low-contrast inherent in NC imaging. We note however that in some capillaries the assumption of constant flow over time is observably false, and thus finding the optimal compromise between robustness and measuring this temporal variability is a subject for further work.

To obtain a single measure of blood cell velocity for each capillary, we must account for the fact that a capillary may appear in multiple segments, or, particularly in the case of abnormally large capillaries, be split between segments. Rather than simply averaging flow measurements between segments, we project them back into the global 
geometry of the whole nailfold, and where they overlap, evaluate which segment produced a more reliable estimate.

One option is to use the residual model-fit from the top-level optimisation, although in practice we found this unreliable. Instead, we note that we do not constrain flow using $V_{s}$ or $V_{\theta}$ and so the method will estimate non-zero flow outside of the vessel, and within the vessel may predict flow directions that do not match the predicted orientation. This provides two measures of flow reliability: firstly, using $V_{s}$ we compute the ratio of mean flow magnitudes inside and outside the vessel; secondly, using $V_{\theta}$ we measure the mean angular difference between the flow direction and orientation at each vessel pixel.

In practice we found that selecting the segment which produced the highest vessel to background flow ratio gave best results, although work to validate measures of flow reliability are ongoing. Having projected estimations of flow from the capillary videos back to the global nailfold geometry, we take the mean flow magnitude over all distal capillaries as a single measure of blood velocity.

\section{Experiments}

\subsection{Data}

112 subjects (50 HC, $12 \mathrm{PRP}, 50 \mathrm{SSc}$ ) were recruited in a tertiary referral centre for SSc and imaged using the new system. Video sequences of all 10 digits (where available) were acquired, generating 1,104 sequences. A total of 23,489 distal capillaries were detected and used to compute measures of capillary density, mean and maximum width, shape, derangement and mean flow velocity. Detecting capillaries takes $\sim 4$ mins per sequence, with processing commencing automatically in the background while live imaging continues. Flow measures are computed offline and take $\sim 1 \mathrm{hr}$ per sequence, processing all capillaries sequentially. If time is a critical factor for analysis, the capillaries may be processed in parallel (e.g. on a GPU), however this was not necessary for this study. For each subject we computed the mean over all their gradeable digits to produce a single value for each parameter ( 8 subjects had one or more ungradeable digits, although all had at least 3 gradeable digits).

\subsection{Results}

Group means and standard errors for each structure and flow parameter are shown in Table 1. Group means for SSc are statistically significantly different to both $\mathrm{HC}$ and PRP for all parameters, with values matching results from earlier studies $[3,4]$. For each individual parameter Fig. 3a shows an ROC curve and $A_{z}$ values for predicting $\mathrm{SSc}$ (positive) versus the combined group HC/PRP (negative) ${ }^{2}$.

We combined the individual parameters in a logistic regression model using HC/PRP vs SSc as a binary output variable, first using only the structural measures and then including flow. Stepwise regression was used to add/remove terms from an initial linear model fit, and in both cases max width (highly correlated with mean width) and

\footnotetext{
${ }^{2}$ HR/PRP are grouped both because we would not expect significant differences in their capillary measures and because detecting SSc is the most clinically relevant task.
} 
(a)

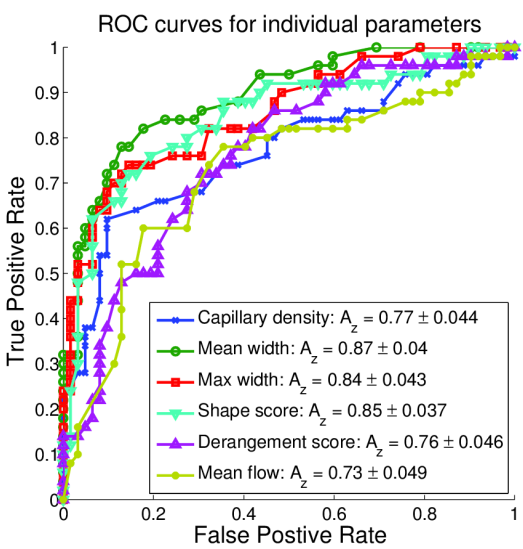

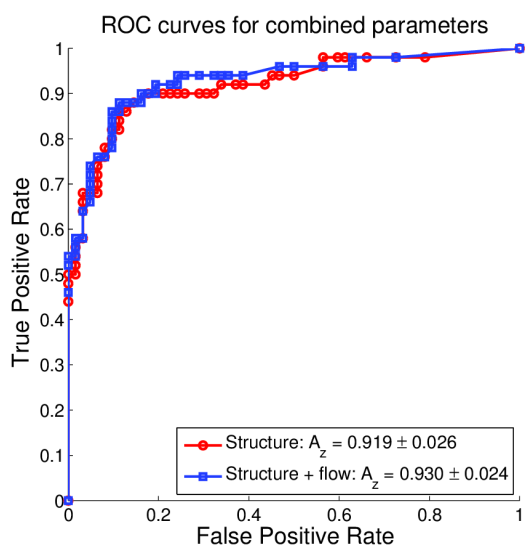

Fig. 3. ROCs for separating HC and PRP from SSc: (a) Indiviudal measures of capillary structure and flow; (b) combined measures in a logistic regression model, with and without flow.

derangement (highly correlated with shape) were discarded. In the latter model flow was retained suggesting it provides additional independent information to the structural measures. To estimate model performance we applied leave-one-out cross-validation to obtain unbiased predictions for each subject (Fig. 3b). The model of structural measures produced an $A_{z}$ of 0.919 , significantly greater than the best single parameter (mean width $\left.A_{z}=0.874\right)$. Including flow further improved performance $\left(A_{z}=0.930\right)$, again indicating that flow contains complementary information for diagnosing SSc.

\section{Conclusions}

We have presented details of a new nailfold capillaroscopy system that we believe enhances the current state-of-the-art of in-vivo microvascular imaging. Carefully selected hardware components, combined with novel, bespoke software, enables fast and easy acquisition of high-quality video sequences in which both capillary structure and blood velocity are measured automatically. We have shown the results of an initial trial of the system in a case/control study in which capillary measures strongly predict patients with SSc. This should now be formally validated against current clinical practice in a prospective study. Blood flow velocity results, while preliminary, are promising and warrant further investigation, and we are currently working on validating the results, including psychovisual tests with human observers and developing a microscopic flow phantom to provide objective ground truth.

To test the sensitivity of flow measures, we are planning trials that include dynamic challenges to induce rapid changes in capillary function. Moreover the optical properties of the system may easily be adapted to assess other measures of capillary function (e.g. using multispectral imaging to assess oxygenation, or UV induced fluorescence to measure oxidative stress), providing an exciting avenue for further investigations of pathophysiology and microangiopathy.

Acknowledgements. This work was funded by the Wellcome Trust. 
Table 1. Group means and standard errors and ROC $A_{z}$ values for each capillary measure. $\sharp, \dagger, \ddagger$ denote significant pair-wise differences for PR vs HC, SSc vs HC, and SSc vs PR respectively.

\begin{tabular}{lcccr}
\hline Parameter & \multicolumn{3}{c}{ Subject group means $( \pm 2$ s.e. $)$} & ROC $A_{z}$ \\
& HC $(\mathrm{n}=50)$ & $\operatorname{PRP}(\mathrm{n}=12)$ & SSc $(\mathrm{n}=50)$ & HC,PRP $v$ SSc \\
$\begin{array}{l}\text { Capillary density } \\
\left(\mathrm{mm}^{-1}\right)\end{array}$ & $6.73 \pm 0.34$ & $7.28 \pm 0.54$ & $5.52 \pm 0.42^{\dagger \ddagger}$ & $0.771 \pm 0.04$ \\
$\begin{array}{l}\text { Mean width } \\
(\mu \mathrm{m})\end{array}$ & $11.8 \pm 0.23$ & $12.7 \pm 0.74$ & $15 \pm 0.71^{\dagger \ddagger}$ & $0.874 \pm 0.04$ \\
$\begin{array}{l}\text { Max width } \\
(\mu \mathrm{m})\end{array}$ & $16.5 \pm 0.91$ & $21.4 \pm 2.94^{\sharp}$ & $27.4 \pm 2.38^{\dagger \ddagger}$ & $0.836 \pm 0.04$ \\
$\begin{array}{l}\text { Shape score } \\
(\in[0,1])\end{array}$ & $0.312 \pm 0.02$ & $0.325 \pm 0.03$ & $0.249 \pm 0.01^{\dagger \ddagger}$ & $0.845 \pm 0.04$ \\
$\begin{array}{l}\text { Derangment score } \\
(\in[0,1])\end{array}$ & $0.649 \pm 0.03$ & $0.689 \pm 0.05$ & $0.54 \pm 0.03^{\dagger \ddagger}$ & $0.761 \pm 0.05$ \\
$\begin{array}{l}\text { Mean flow velocity } \\
\left.(\mathrm{mm} \mathrm{s})^{-1}\right)\end{array}$ & $0.311 \pm 0.05$ & $0.383 \pm 0.10$ & $0.235 \pm 0.04^{\dagger \ddagger}$ & $0.726 \pm 0.05$ \\
\hline
\end{tabular}

\section{References}

1. Herrick, A.L.: Contemporary management of raynaud's phenomenon and digital ischaemic complications. Current Opinion in Rheumatology: 23 (November 2011) 555-561

2. Cutolo, M., Pizzorni, C., Secchi, M.E., Sulli, A.: Capillaroscopy. Best Practice and Research Clinical Rheumatology 22(6) (2008) 1093-1108

3. Murray, A.K., Moore, T.L., Manning, J.B., Taylor, C., Griffiths, C.E.M., Herrick, A.L.: Noninvasive imaging techniques in the assessment of scleroderma spectrum disorders. Arthritis Rheum 61(8) (2009) 1103-11

4. Berks, M., Tresadern, P., Dinsdale, G., Murray, A., Moore, T., Herrick, A., Taylor, C.: An automated system for detecting and measuring nailfold capillaries. In Golland, P., Hata, N., Barillot, C., Hornegger, J., Howe, R., eds.: MICCAI 2014. Volume 6533 of Lecture Notes in Computer Science., Springer Berlin Heidelberg (2014) 658-665

5. Brox, T., Bruhn, A., Papenberg, N., Weickert, J.: High Accuracy Optical Flow Estimation Based on a Theory for Warping. In: Computer Vision - ECCV 2004. Springer Berlin Heidelberg, Berlin, Heidelberg (2004) 25-36

6. Mawson, D.M., Shaw, A.C.: Comparison of CapiFlow and frame by frame analysis for the assessment of capillary red blood cell velocity. J Medica Eng Tech 22(2) (1998) 53-63

7. Shih, T.C., Zhang, G., Wu, C.C., Hsiao, H.D., Wu, T.H., Lin, K.P., Huang, T.C.: Hemodynamic analysis of capillary in finger nail-fold using computational fluid dynamics and image estimation. Microvascular Research 81(1) (2011) 68 - 72

8. Dobbe, J.G.G., Streekstra, G.J., Atasever, B., Zijderveld, R., Ince, C.: Measurement of functional microcirculatory geometry and velocity distributions using automated image analysis. Medical \& Biological Engineering \& Computing 46(7) (2008) 659-670

9. Allen, P.D., Taylor, C.J., Herrick, A.L., Moore, T.: Image analysis of nailfold capillary patterns from video sequences. In Taylor, C., Colchester, A., eds.: MICCAI'99, Berlin, Heidelberg, Springer Berlin Heidelberg (1999) 698-705

10. Horn, B.K.P., Schunk, B.G.: Determining optical flow. Artif. Intell. 17 (August 1981) 185203 